\title{
PRACTICAL ASPECTS REGARDING THE RELIEF OF BACK PAIN WITH THE THERMAL MASSAGE BED
}

\author{
Stan Zenovia ${ }^{1}$, Bastiurea Eugen ${ }^{2}$ \\ ${ }^{1,2}$ Human Performance Research Centre, Faculty of Physical Education and Sport, \\ "Dunarea de Jos" University of Galati, Romania \\ zenovia.stan@ugal.ro \\ eugen.bastiurea@ugal.ro
}

\begin{abstract}
The beneficial effects of massage beds and massage chairs are well known. They are more and more complex but also more accessible for the population. In this study we showed the possibility of introducing the Ceragem massage bed in multidisciplinary programs for pain relief and recovery from back diseases. Although massage beds are frequently used all over the world, they are generally used only for relaxation and there are not many specialized studies that provide details about concrete ways of their use. In this study we presented some general practical aspects regarding the use of this device and a synthesis of its efficiency of integration in physical activity sessions. We made observations during 40 months (2017-2020) over 3600 sessions in which we used the massage bed. These conclusions must be accepted with caution, because they concern only this group of subjects and the study carried out in the specific conditions of adapted motor activities.
\end{abstract}

Keywords: Back diseases, elongation of the spine, prophylaxis

\section{Introduction}

Currently Ceragem sells massage beds in about 80 countries. Since 2010, the Korean company has even marketed a luxury model, $\mathrm{C}$, which "incorporates massage and CERAGEM PRESSURE while radiant heat with far infrared is optimized using jade and an epoxy carbon panel".

Recent studies showed that massage is effective in reducing back pain, while heat during massage increases a person's sense of well-being. Ceragem thermal massage bed uses the principles of thermal massage to provide a therapeutic tool for people with medical conditions but it is also a means of prophylaxis for these problems. We noticed a significant reduction in reported pain in the thoracic and lumbosacral areas. Thermal massage with jade increases the production of white blood cells by promoting the rejuvenation of cells in the body. As a result, the body's immune system is stronger and more active. It ensures the health of the musculoskeletal system, metabolism, nervous system and all vital organs of the body. Spinal decompression is performed (A. Chohan et al. in 2014). The efficiency of using this massage bed in pain relief was also demonstrated by Stan Z. in 2019. 
The effectiveness of using this device in relation to manual massage is emphasized by Zuzana Heinzovaa, Juraj Borovskya and Petr Pelcb in 2019. Following a study by Lee YH he all in 2011, it turned out that massage and heat applied with a massage bed, 40 minutes a day, in 10 sessions (assessing heart rate variability, sympathetic skin response and serum levels of cortisol and norepinephrine) resulted in relaxation of autonomic nervous system without side effects. In the case of post herniated disc diseases, by restoring the normal position of the vertebrae, a massage bed becomes a powerful tool in the fight against the hernia itself and its unpleasant consequences. The massage rollers roll over the entire dorsal area and gently stretch the spine. This eliminates the movement of the vertebrae, pinching and pressure on the nerve endings thus revitalizing the nervous system as a whole. The intervertebral cartilage and discs are restored, the symmetry of the pelvic bones and humeral returns, while infrared heating suppresses inflammation, stimulates blood circulation and metabolism (budivel.ru/)

In 2010, Marienke van Middelkoop et al., studied the application of multidisciplinary treatment (transcutaneous electrical nerve stimulation, low-level laser therapy, education, massage, behavioural treatment, traction, compared to behavioural treatment). It has been found that multidisciplinary treatment reduces pain intensity and short-term disability) and that degeneration of the vertebrae and discs and misalignment of the spine is considered to be the cause of many health problems. When the spine is deformed, the intervertebral spaces change and can cause pressure on the nerves. It is possible that some treatments, performed slowly by certain mechanical devices may have a very good effect on the rearrangement of the vertebrae. At the same time, it can help relieve muscle pain, correct body posture, promote blood circulation, improve muscle stiffness, etc. (drhealthbenefits.com)

\section{Methods}

The study includes observations made in over 3600 sessions in which we used the massage bed, during 2017-2020 (approximately 40 months). We selected 64 adult subjects who followed the program of adapted motor activities for at least 18 months. At the beginning of the program, they performed 3 sessions per week and, depending on the condition and the progress registered, we set only one 90 min session per week for maintenance. We didn't include in this study people who performed a smaller number of sessions, who had conditions that were within the prohibitions of the device, children and those who did not want to include this method in their training program. The use of this method of recovery and relaxation is closely related to exercise programs that have the role of stabilizing the alignment of the spine in terms of musclejoint. The massage bed is very effective only together with a customized program, with physical exercises to maintain the correct positions during elongations and traction performed by it. In 
addition to physical exercises, acupressure, inversion bed, vibromassage, TENS, analgesic stations, isokinetic bands, medical orthotic insoles or electroacupuncture can be used in the same time.

\section{CERAGEM MASSAGE BED MB - 1101 (ro.ceragem.com)}

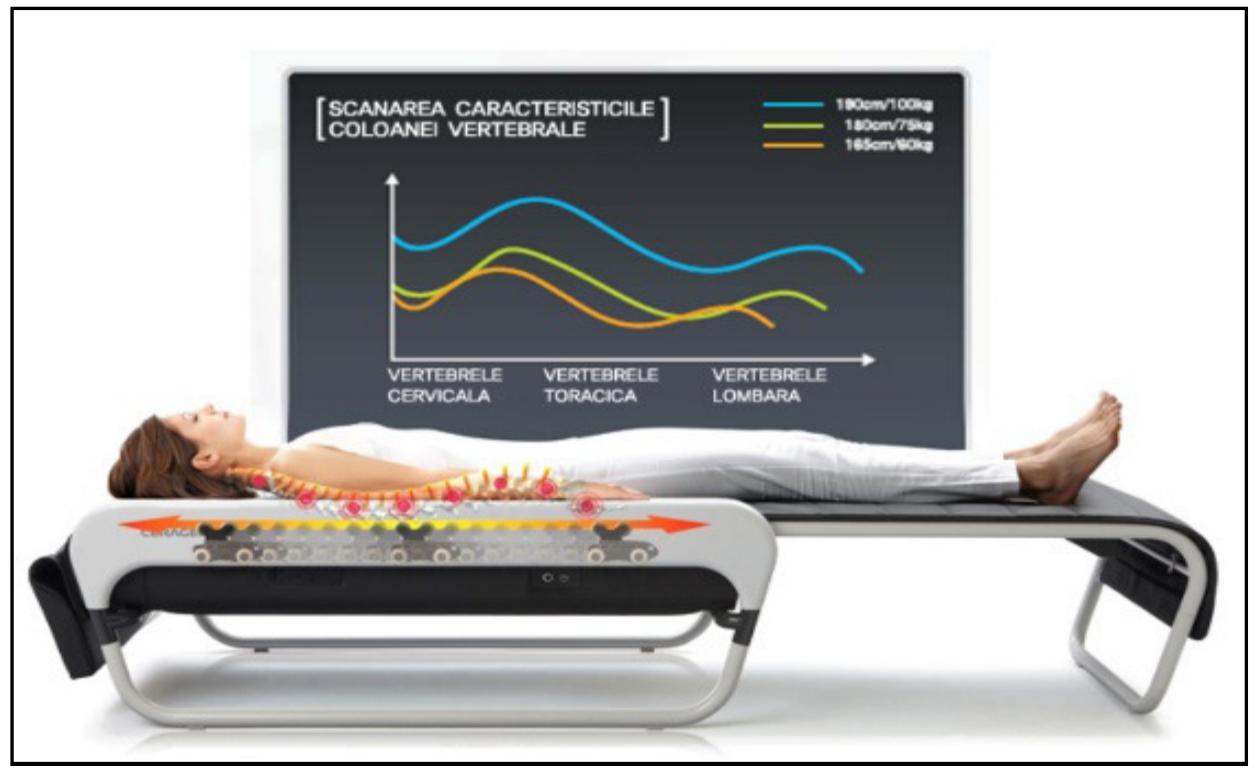

Figure 1. Ceragem Massage Bed

The bed's internal projector moves along the spine from the cervix to the pelvis and analyses the length and curves of the spine to perform a proper body massage. The internal projector descends from the cervical vertebrae and reaches the coccyx to stimulate the different points of application of the spine in each of us, namely the points of application located on the muscles that support the vertebrae (figure 1). 


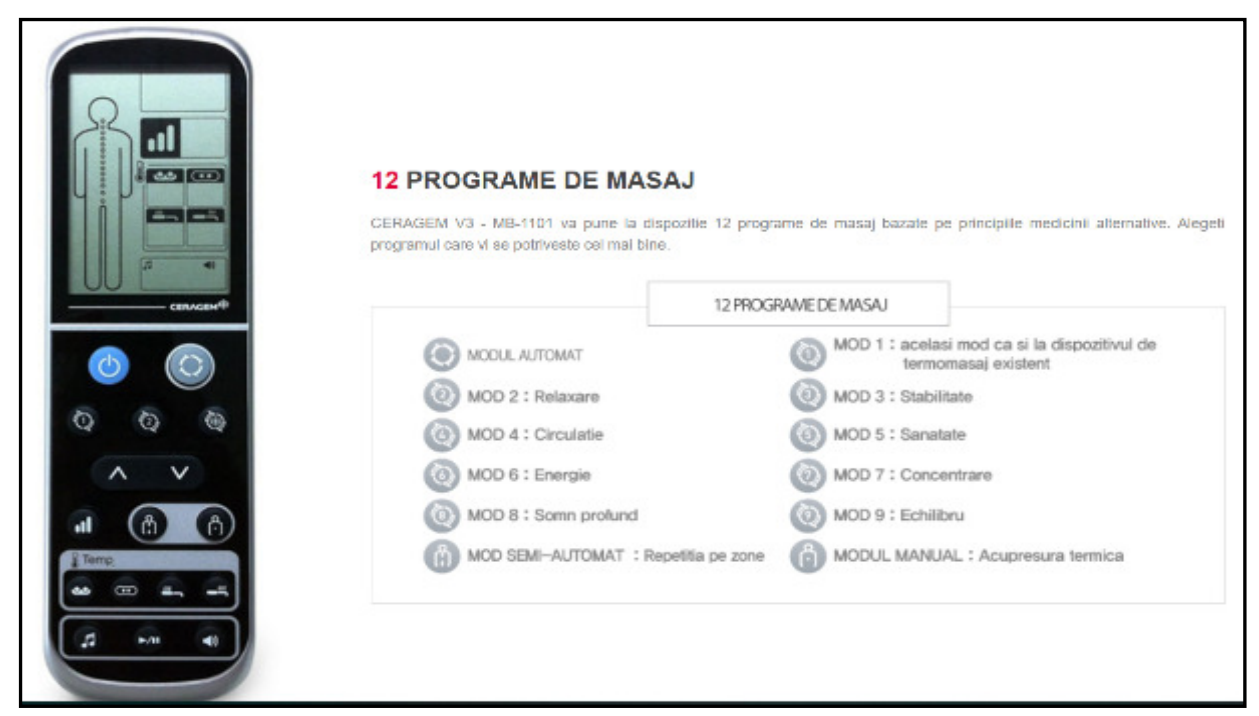

Figure 2. Programs accessible thru the remote control

Figure 2 shows the accessible programs: Relaxation, Circulation, Energy, Deep Sleep, Stability, Health, Concentration, Balance, Manual, Semi-automatic. Spine scanning is performed as the internal projector moves along the body from head to pelvis while the length of the spine and the degree of curvature of the spine are measured to suggest a massage that best fits your body. The curves of each subject using the bed are measured. Working between the cervical vertebrae and the coccygeal vertebrae, the internal projector also stimulates points along the spine that vary from person to person, especially the pressure points in the muscles on the sides of each vertebra. (www.bodycurehealth.com)

\section{Results and Discussions}

Body pain and especially back pain is a result of the following conditions of the subjects: dehydrated intervertebral discs; compressed intervertebral spaces; intraspongios hernias in the dorsal area; disc protrusions in the dorsal and lumbar area; post herniated disc recovery in the lumbar area; pain in the sacral and coccygeal area; pain in the knee area (internal and posterior) in the lower limb (usually in the MI opposite the right arm) associated with pain in L5-S1 on the same side; pain and contractions in the calves; heel pain; pain in the scapular girdle and in the MS, bursitis in the scapular girdle, etc. After the complete evaluation, we established a program of adapted motor activities. Next, we presented the general structure of a program and the practical way in which the massage bed was integrated in close correlation with the other methods. We also listed the stages and the way in which each stage was applied as well as the percentage covered by each activity. 
Stage 1. Evaluation of the health condition at that moment, synthesis of information from medical doctors and other therapists, recovery methods used until that moment, results obtained and motor and functional evaluation performed in the room.

Stage 2. In the first sessions, after the evaluation stage, we established the program of adapted motor activities. According to the health condition, out of the 64 people, we included the massage bed as follows (diagram 1): at 34\% after 1-2 sessions; at 12\% after 3-4 sessions and at $54 \%$ we introduced the bed right from the first session, under certain conditions.

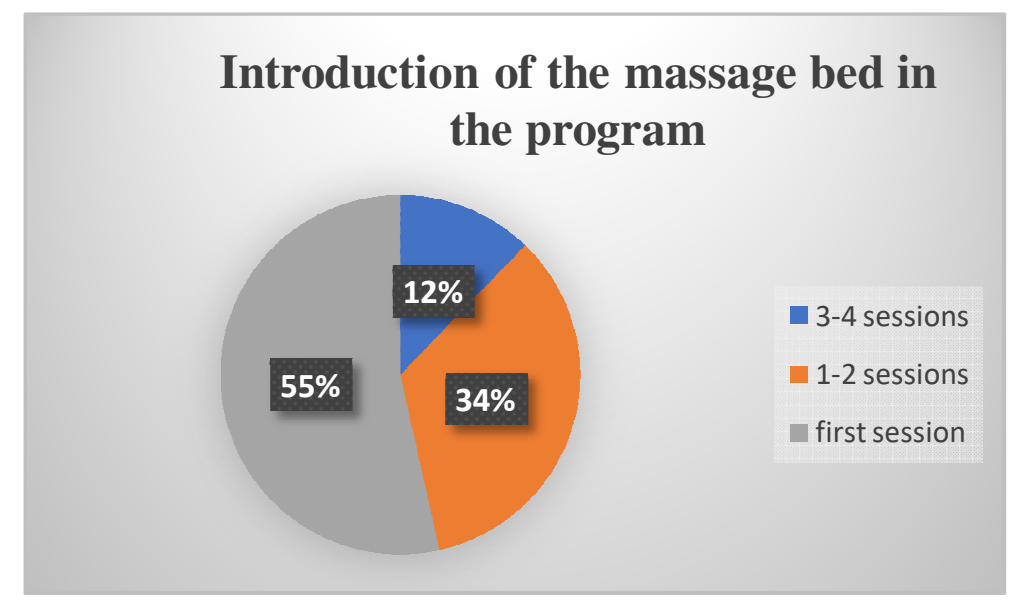

Diagram 1. Introduction of the massage table in the program

Step 3. Once we established if the subject has no prohibitions to perform this type of massage (there are prohibitions very clearly specified in the technical manual of the device), we assessed of how it supports this type of massage. Depending on the rigidity of the spine, the pain perceived during the pressure of the jade rollers on the muscles, the muscle-joint elasticity, etc., we established under what conditions the available automatic massage programs was performed.

For this purpose, we first adjust the pressure, using the manual control from the remote control. If the physical impact was too high, we placed over the trolley roller a blanket folded in 2 or even in 4 . We avoided the painful areas, set the sensitive areas and pain radiation points in case of compressed nerves in the intervertebral spaces.

The duration of this first direct contact with the massage rollers was between 10 and 20 minutes. We perform the manual directed massage at level 1 of intensity so that the impact is as small as possible. In $56 \%$ of the subjects, only one manual directed session was needed, in $14 \%$ 2 sessions were needed and in 30\% a period of body accommodation in 4-5 sessions was needed (diagram 2). 


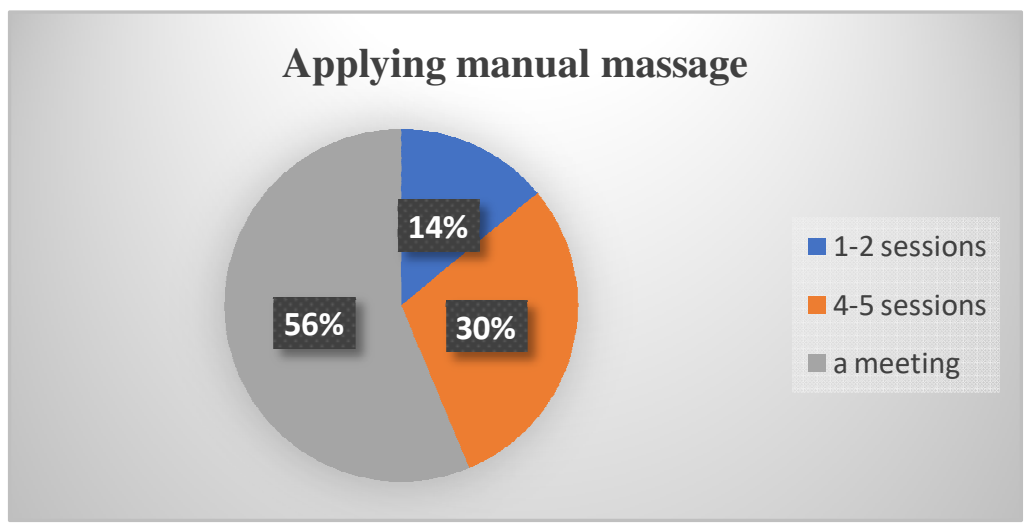

Diagram 2. Applying the manually directed massage, level 1 of intensity

Step 4. In the following sessions, we applied the short automatic massage program of 18 minutes. It is a relaxation program and the massage rollers do not perform acupressure. They just massage the paravertebral muscles and elongate the spine, area by area. It starts with level 1 intensity. Diagram 3 shows that $59 \%$ of those subjects needed 1 or 2 short sessions and $41 \%$ needed 3-4 sessions. These short automatic massage sessions, without putting high pressure on the curves of the spine, are performed in any of the other stages when the subject is in a more delicate health condition. At any time, the massage on this bed can be excluded if the situation requires it.

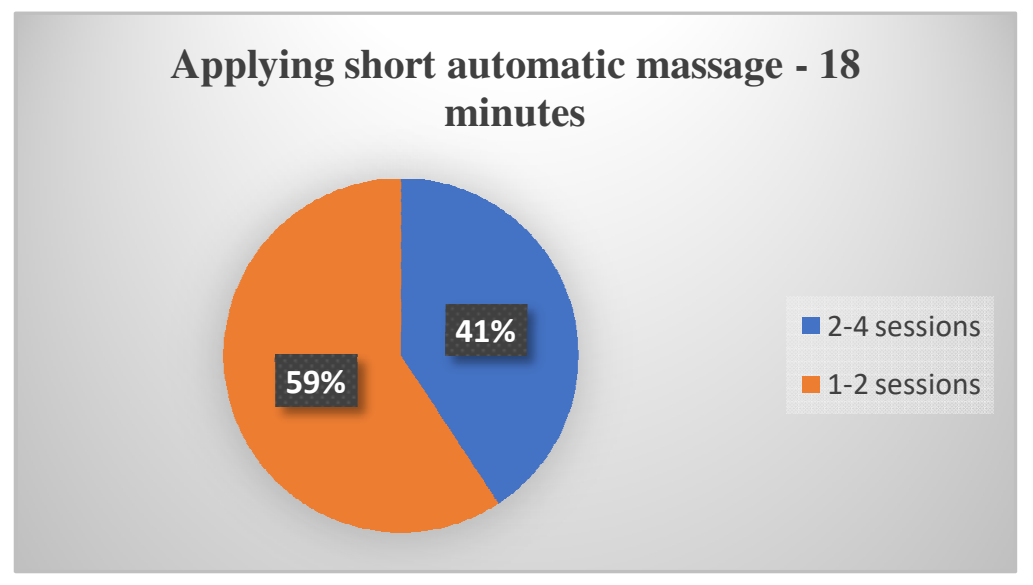

Diagram 3. Application of the short automatic program (18 minutes)

Stage 5. In diagram 4 we observed how many sessions were needed at intensity level 1. The programs were individualized, they were at the maximum duration (36 minutes,), heat and acupressure were applied (depending on prohibitions). Thus, only a small percentage (5\%) needed only 1-2 sessions, 13\% 5-6 sessions, 39\% 10 sessions and 43\% needed $15-25$ sessions before reaching the level 2 intensity. 


\section{Applying the automatic massage at the} first level of intensity - 36 minutes

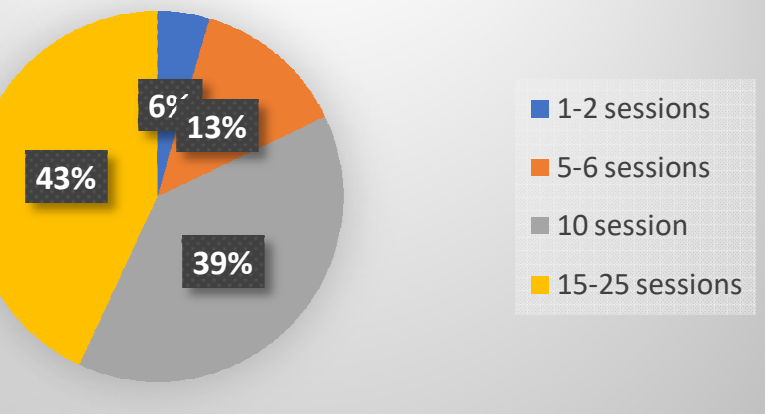

Diagram 4. Application of individualized massage, complete program, level 1

Stage 6. The advanced stage. Diagram 5 shows what percentage of those observed reached the maximum level of this device (level 6). Thus, it is observed that $42 \%$ did not reach the last level, 39\% reached level 6 after $80-90$ sessions and only 19\% in a relatively short time (30-50 sessions).

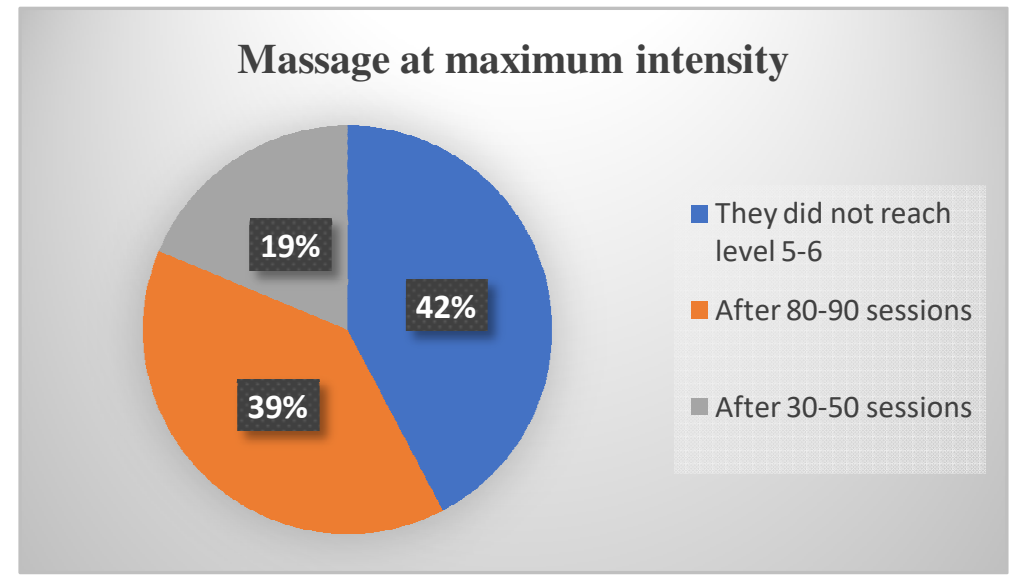

Diagram 5. Applying the complete massage at maximum intensity level

These stages are a result of our practical activity and each of them represents only a part of the $\quad 90$ minute session. It is not effective to apply this type of massage without support with individualized exercise programs, except for healthy people who want to perform a complete relaxation session. Any correction of the curves of the spine with this massage only, is maintained just for a short period. The deficient position of the body is installed in time and it takes patience and a lot of work to correct as efficiently as possible everything that needs to be corrected. Since 2000, Jang Jun-Hyuk et al., Have demonstrated the effectiveness of this system of massage and elongation of the spine, with effects on pain in the cervical area and the entire 
posterior area. They also observed effects on mental stability and recovery from fatigue. C. So et al. in 2007, highlights the effect of thermomechanical massage on the function of human immune cells. Contrary to these studies, there have been observations that there may be side effects on those who use this massage system (www.chinacsr.com). Because there have been several incidents in Romania, it is very important that those who use this device do not forget that it has enough contraindications and that they must be strictly observed.

\section{Conclusions}

The results of this study can help specialists who want to use this type of massage, elongation and pressure on the spine. We noticed that a long period of time is necessary for a body to restore the correct alignment of the vertebrae, to increase its musculoskeletal mobility so that the massage can be performed at maximum intensity (80-90 sessions). The use of this very useful device must be made respecting all the rules required by the manufacturer and in very strict relation to the real possibilities of acceptance of the subjects. In this study we highlighted the importance of designing a detailed program for applying this massage and using exercise programs in parallel. The results are perceived quickly, sometimes even from the first session, but they have no effect without physical support. Those who want to restore their posture, to improve certain conditions caused by the pressure of the vertebrae on the nerves, must allocate enough time and complete the work at the gym with a minimum program of physical activity at home. In future studies we can detail each model of improvement with the help of the massage bed of the various deficiencies mentioned in this study.

\section{Bibliography}

1. A. Chohan, H. Roddam, S. Deakins, S. Islam, J. Selfe. 2014. Determining the effectiveness of the ceragem V3 automatic thermal massage bed for back pain. The Journal of Bone and Joint Surgery 96-B(4):43.

2. C. So, J. Sarath, R. Giolli, S. Gollapudi. 2007. The Effect Of Thermal Massage On Human T-Lymphocyte And Natural Killer Cell Function. The Internet Journal of Alternative Medicine. Volume 6 Number 1.

3. Jang Jun-Hyuk, Kim Kyung-Ho, Kim Jang-Hyun. 2000. Effect of the Ceragem Master Heat Bed for Posterior Neck Pain and Low Back Pain, The Journal of Pediatrics of Korean Medicine 14(2).

4. Lee YH, Park BN, Kim SH. 2011. The effects of heat and massage application on autonomic nervous system. Yonsei Med J., Nov;52(6):982-9.

5. Marienke van Middelkoop M, Rubinstein SM, Kuijpers T, Verhagen AP, Ostelo R, Koes BW, Van Tulder MW. 2011. A systematic review on the effectiveness of physical and rehabilitation interventions for chronic nonspecific low back pain. Eur Spine J., Jan;20(1):19-39. 
6. Stan Zenovia. 2019. Combined method of improving the quality of life by relieving the back pain. International Journal of Current Research (IJCR), Volume 11, Issue 01, January, DOI 10.24941/ijcr.33689.

7. Zuzana Heinzovaa, Juraj Borovskya and Petr Pelcb. 2014. Financial, time and efficiency assessment of physiotherapeutic bed and manual massage techniques. Juraj Borovskya and Petr Pelcb. Faculty of Biomedical Engineering. Czech Technical University in Prague. Czech Republic. K. Nemocnici 2014/4 Kladno.

8. $\quad$ http://www.bodycurehealth.com/Bed_V3+.php

9. https://budivel.ru/en/banya/massazhnaya-krovat-protivopokazaniya-i-pokazaniya-nefritovye-krovati.html

10. https://www.chinacsr.com/en/2007/03/13/1122-ceragem-massagers-reportedly-causing-adverse-reactions/

11. $\quad$ https://drhealthbenefits.com/food-bevarages/fruits/benefits-of-java-plum 